\section{PHOTOGRAPHY WITH THE ELECTRON MICROSCOPE}

A MEETING was held on November 25 at the Royal Photographic Society's rooms in London to discuss the use of photographic materials in the electron microscope. The meeting was arranged jointly by the Scientific and Technical Group of the Royal Photographic Society and the Association for Scientific Photography.

The first speaker, Mr. G. Parr, gave a brief description of the principles of the electron microscope based upon analogy with the well-known optical microscope. Mr. L. V. Chilton, of Ilford, Ltd., followed with a paper written jointly by himself, Dr. E. M. Crooks and Dr. F. M. L. Sheffield, both of the Rothamsted Experimental Station, dealing with the behaviour of a range of photographic materials in the R.C.A. electron microscope Type B. The meeting was concluded by Dr. D. G. Drummond, of the British Cotton Industry Research Association, who showed a number of examples of the use of the electron microscope in cotton research.

Apart from early sporadic uses of photographic materials to record the variations of energies in electron beams, probably the first industrial use was in the continuously evacuated cathode ray tube. The type of tube employing a fluorescent screen is, however, much more popular, and while special problems occur, the behaviour of the photographic material is not dissimilar from its behaviour under ordinary conditions of light exposure. The photographically effective light emitted by the screen is usually blue or green, so that no abnormal problem arises in colour sensitization. A camera of the conventional type is usually used, and the only significant divergence from standard practice is the extreme shortness of exposure time which is inevitable in the photography of rapid transient phenomena. Exposure times may be as short as 0.00001 sec., and under these conditions the relative speeds of two materials may be reversed when compared with their relative speeds as measured at an exposure time of, say, 0.01 sec.

Little work seems to have been published on the behaviour of different photographic materials to the electron beam of the continuously evacuated cathode ray tube, and with the development of the electron microscope such an investigation has become more than ever necessary. A valuable contribution has been made by the authors of the joint paper read by Mr. Chilton, in which the results of tests carried out on a range of Ilford plates are described. Immediate application of the results was required for the photography of plant viruses, which give very poor contrast; and in consequence the range of materials tested was chosen for their relatively high contrast as normally measured by exposure to light.

It would be expected a priori that the density produced on a photographic material would depend upon the beam current and the accelerating potential. The beam current is analogous to the intensity of light and the accelerating potential to the colour. Exposures were made over a range of beam currents, and the logarithm of the value plotted against the density produced yields a characteristic curve for a given accelerating potential. By varying the accelerating voltage, a family of curves can be obtained which should show in what manner speed and contrast vary with voltage for each material. A difficulty arises which is peculiar to electron microscope technique; as the voltage is changed, the focal lengths of the 'lenses' change and this alters the current density. The correction is calculable, but the authors used a method originally due to the National Physical Laboratory in which the brightness of the image as seen on the willemite viewing screen is compared with a comparison patch of light projected adjacent to the sereen through one of the viewing ports. This enables the magnification to be altered as the kilovoltage is altered to maintain equal visual activation of the screen for a constant beam current.

Several interesting features were revealed by the results. A voltage-range from 15 to $60 \mathrm{kv}$. was explored, and in all the plates tested there was a marked tendency for the contrast to rise as the kilovoltage was increased. Over the range examined the relation between kilovoltage and contrast was linear, though in some cases there was an indication that at high kilovoltages a limiting contrast was being approached, as would be expected. At low kilovoltages there is a tendency for the characteristic curve to decrease in contrast at medium densities as if a relatively low maximum density was being approached. Because the contrast increases with voltage, it must not be assumed that high kilovoltages should be used for objects showing low contrast. The effective contrast of the object may itself be dependent on voltage in much the same way that an object exhibits lower contrast the higher the voltage used in the tube in radiography. The analogy cannot be pushed too far and is only given as an example. The behaviour of objects under electron bombardment may vary, so no general principles can at present be formulated, though visual observation of the willemite screen suggested in the case of plant viruses that there is a small increase of contrast with voltage. It is suggested that the decrease of contrast at lower kilovoltages is caused by the increased absorption of electrons in the outermost layer of gelatin in the photographic plate, an absorption. which will be more effective the lower the energy of the electron beam. This theory suggests that the special Schumann plates in which the silver bromide crystals project through the gelatin surface (probably with a very thin covering layer of gelatin) might be worthy of trial. These plates are used for ultraviolet spectroscopy in the region where gelatin has a very high absorption.

A comparison of the relative speeds of the various photographic plates examined also yields interesting data. The exposure time given in the electron microscope was 10 sec., this figure being chosen because of the difficulty of manipulating the shutter to obtain reproducible shorter exposures. At the same time serious difficulty was experienced in maintaining constant beam current during exposures due to mains fluctuations. Both these matters might well receive attention in the design of future instruments. In addition to the electron exposures, all materials were exposed to light of various intensities for $10 \mathrm{sec}$. under normal sensitometric conditions to obtain characteristic curves to light for comparison.

At any given kilovoltage the order of relative speeds, as measured by the exposure necessary to produce a density of $1 \cdot 0,2 \cdot 0$ or $3 \cdot 0$, was roughly the same for electrons as for light, except in one instance in which the sensitivity to light was substantially augmented by colour sensitization. The total range 
of sensitivities to electrons was much compressed compared with the range to light. At a density of $2 \cdot 0$ the total range of speeds to light was 100 to 1 , whereas to the electron beam at $45 \mathrm{kv}$. it was only 13 to 1 . This is not surprising when it is remembered that the photographic materials in question were made primarily for exposure to light. Quite apart from colour sensitivity, the speed of a photographic emulsion to light depends on a number of factors. In general, the larger the silver bromide grains in an emulsion the greater the speed; but even at constant grain-size a wide range of speeds can be produced by the technique employed in making the emulsion. According to modern theory, the primary action of radiation in latent image formation is the release of electrons within the silver bromide crystals. Apart from the possibility of recombination, these electrons fall into 'traps' which become charged and can then hold and neutralize the mobile silver ions, thus forming specks of metallic silver in their immediate neighbourhood. When the specks have grown to a certain minimum size the grain is rendered developable. It is believed that the initial size of the specks or electron traps is determined by the emulsionmaking technique; the larger the specks, provided they are not sufficiently large to render the grain developable spontaneously, the greater the sensitivity to light. A delicate balance in all grains is not possible, so if few are to be spontaneously developable, the majority will require a significant number of added silver atoms to raise them to the necessary size. It is therefore probable that the absorption of a number of light quanta is necessary to render a grain developable, and control of sensitivity can be effected over a wide range by adjustment of specksizes during making. When the same emulsions are exposed to X-rays or high-velocity electrons, the energy of the quanta being many thousands of times greater, it may be that the absorption of a single quantum is sufficient to provide enough electrons to enable even relatively insensitive specks to reach dimensions sufficient to render the grain developable.

This argument is necessarily only given in brief, but it suggests that sensitivity to light is much more dependent on the condition of the sensitive specks than is sensitivity to electrons or X-rays. Sensitivity to high-energy quanta would be expected to depend on the probability of a quantum being absorbed, and thus more on grain size. It is indeed possible to produce emulsions relatively very sensitive to $\mathrm{X}$-rays and at the same time relatively insensitive to light, and such materials are marketed for use without fluorescent screens.

Returning to the results communicated by $\mathrm{Mr}$. Chilton, he and his co-authors endeavoured to find the relation between photographic speed and kilovoltage of the electrons. This was complicated by the fact that the reference standard between the different kilovoltages was the brightness of the willemite viewing screen. Some idea of the efficiency of the screen in converting electron energy into light energy was obtained, and it was then possible to make a rough correction to the exposure axis so that direct comparison of speed at different kilovoltages was possible. This revealed that the variation with kilovoltage of speed as measured by the density produced by an arbitrary standard exposure was different for the various plates examined. In some there was little change over the range of kilovoltages used, in others the photographic speed increased with kilo- voltage and reached a maximum near the upper limit of the range, and again in others there was a steady rise over the whole range.

These results show clearly the necessity for a fuller investigation, and it is to be hoped that the authors will be able to extend their work to cover a wider range of materials and conditions. Other workers have published results of a similar nature, some of which are in general agreement with the results discussed here. Substantial disagreement between results obtained by different workers is not unusual where photographic materials are concerned and should not be taken to indicate anything but the uniqueness of every photographic emulsion type. Special mention may be made of work published by von Borries (Physikal. Z., 43, 190 ; 1942) using Agfa and Perutz plates, which shows substantial qualitative agreement in all points with the Ilford plates used in the tests described.

There is no doubt that there is much to be learned about the technique of electron micrography of different subjects, and the selection of the most suitable photographic material and its treatment. It must also be remembered that manufacturers have not yet made any attempt (or at any rate no outwardly apparent attempt) to make plates specially suited to the needs of electron microscopy. Such a development may be expected when more is known of the requirements and a demand is made for special materials, just as has occurred in the case of $\mathrm{X}$-rays, and more recently in the photography of the cathode ray tube screen.

The authors of the papers who contributed to a well-attended and most successful afternoon meeting are to be congratulated on arousing interest in such an important subject, and making so valuable a contribution, which it is hoped will whet the appetite of other workers in the field who are fortunate enough to have the necessary equipment for further investigations. The full text of the papers is to be published in the Photographic Journal early this year.

\section{STRUCTURE OF DIAMOND}

$\mathrm{D}$ IAMOND is generally thought to be one of the most perfect crystals occurring naturally: its atomic structure was first investigated in the very early days of X-ray crystal analysis. It turned out that the atoms of the crystal were arranged on two interpenetrating face-centred cubic lattices, the corner of one cube lying one quarter of the way along the cube diagonal of the other. Whether the resulting crystal possessed full octahedral symmetry or was of the lower tetrahedral type, a question on which crystallographers were divided, could not be settled by the additional X-ray evidence. Some ten years ago, new interest in the structure of diamond was aroused by the work of Robertson, Fox and Martin ${ }^{1}$, who showed that the infra-red absorption and ultraviolet transparency of diamonds placed them in two classes. Of the two types, the first and commoner was opaque to both infra-red and ultra-violet radiation, while the second, Type II, is rare and is transparent to these radiations. The more recent $\mathrm{X}$-ray investigations of Lonsdale and Smith ${ }^{2}$ have shown that although the two types are structurally identical on an atomic scale, there are abnormalities in the diffraction patterns of Type I diamonds which suggest some kind of 'mosaic' structural difference in the two 